\title{
Source localization of the Nogo-N2: a developmental study
}

Citation for published version (APA):

Jonkman, L. M., Sniedt, F. L., \& Kemner, C. (2007). Source localization of the Nogo-N2: a developmental study. Clinical Neurophysiology, 118(5), 1069-77. https://doi.org/10.1016/j.clinph.2007.01.017

Document status and date:

Published: 01/01/2007

DOI:

10.1016/j.clinph.2007.01.017

Document Version:

Publisher's PDF, also known as Version of record

Document license:

Taverne

Please check the document version of this publication:

- A submitted manuscript is the version of the article upon submission and before peer-review. There can be important differences between the submitted version and the official published version of record.

People interested in the research are advised to contact the author for the final version of the publication, or visit the DOI to the publisher's website.

- The final author version and the galley proof are versions of the publication after peer review.

- The final published version features the final layout of the paper including the volume, issue and page numbers.

Link to publication

\footnotetext{
General rights rights.

- You may freely distribute the URL identifying the publication in the public portal. please follow below link for the End User Agreement:

www.umlib.nl/taverne-license

Take down policy

If you believe that this document breaches copyright please contact us at:

repository@maastrichtuniversity.nl

providing details and we will investigate your claim.
}

Copyright and moral rights for the publications made accessible in the public portal are retained by the authors and/or other copyright owners and it is a condition of accessing publications that users recognise and abide by the legal requirements associated with these

- Users may download and print one copy of any publication from the public portal for the purpose of private study or research.

- You may not further distribute the material or use it for any profit-making activity or commercial gain

If the publication is distributed under the terms of Article $25 \mathrm{fa}$ of the Dutch Copyright Act, indicated by the "Taverne" license above, 


\title{
Source localization of the Nogo-N2: A developmental study
}

\author{
L.M. Jonkman ${ }^{\mathrm{a}, *}$, F.L.F. Sniedt ${ }^{\mathrm{a}}$, C. Kemner ${ }^{\mathrm{a}, \mathrm{b}}$ \\ a Department of Neurocognition, Faculty of Psychology, Maastricht University, P.O. Box 616, 6200 MD Maastricht, The Netherlands \\ ${ }^{\mathrm{b}}$ Department of Child and Adolescent Psychiatry, University Medical Centre, Utrecht, The Netherlands
}

Accepted 28 January 2007

\begin{abstract}
Objective: The aim of the present study was to investigate whether similar neural sources are involved in generating Nogo-N2 scalp topography in children and adults.

Methods: Source analysis was performed on the Nogo-N2 data from two groups of children (sixteen 6/7 year-olds and seventeen 9/10 year-olds) and seventeen young adults (aged 19-23 years) that were obtained in a prior study by Jonkman LM. The development of preparation, conflict monitoring and inhibition from early childhood to young adulthood; a go/nogo ERP study. Brain Res 2006;1097:181-93.

Results: In both children and adults a bilateral source pair in the medial frontal cortex (near ACC) was involved in the generation of Nogo-N2 activity. However, children needed an additional, posteriorly located source pair to adequately explain the Nogo-N2 distribution. In 6/7 year-olds this posterior source was localized in occipito-temporal areas, whereas in $9 / 10$ year-olds the posterior sources shifted to parietal locations.

Conclusions: Although children recruit similar frontal regions as adults in the Nogo-N2 time window, the additional activation of posterior sources might indicate that early executive control performance is less automatic or requires more effortful control in children. This in turn might cause them to rely on more basic stimulus processing or to activate additional attention-related areas.

Significance: Results from the present study show that in children, a more diffuse brain network is involved in executive control processing (conflict monitoring) in the Nogo-N2 time window than in adults.

(c) 2007 International Federation of Clinical Neurophysiology. Published by Elsevier Ireland Ltd. All rights reserved.
\end{abstract}

Keywords: Child development; Conflict monitoring; Dipole modeling; Nogo-N2; Anterior cingulate cortex

\section{Introduction}

The present study is aimed at elucidating which brain sources underlie the development of the Nogo-N2 eventrelated brain potential (ERP) component, by applying source analyses methods to the data from an earlier paper by Jonkman (2006). In the latter paper we compared the performance and ERPs of 6-7 and 9-10 year-old children and young adults during performance of a Go-Nogo (cued CPT-AX) task. In this task the subjects are presented with a series of letters and have to respond to the letter X (target), but only when it is preceded by the letter A (cue), this

\footnotetext{
* Corresponding author. Tel.: +31 43 3881956; fax: +31 433884125 .

E-mail address: L.Jonkman@psychology.unimaas.nl (L.M. Jonkman).
}

is the Go condition. In situations in which the cue letter A is not followed by the target stimulus (Nogo condition), the response that has been prepared during the cue-probe interval has to be inhibited. In the Nogo-condition (compared to Go) an ERP response consisting of a negativity (Nogo-N2) followed by a positivity (Nogo-P3) above fronto-central cortex is evoked. In adults the Nogo-N2 has been linked to the process of response inhibition in some studies (Falkenstein et al., 1999; Fallgatter and Strik, 1999; Jodo and Kayama, 1992), whereas in later studies (Donkers and Van Boxtel, 2004; Nieuwenhuis et al., 2003; Van Veen and Carter, 2002a) the negativity was linked to the process of conflict monitoring. In the prior developmental study by Jonkman (2006) it was concluded on the basis of performance measures that attention processing in the CPT-AX task improved most strongly 
between the age of 6 and 10, whereas inhibitory behavior started to improve later, after the age of 10 . Nogo-N2 effects were, however, found to be largest in the youngest (6- to 7-year-old) children and decreased linearly with age. On the basis of these and other ERP responses in reaction to the cue, it was concluded that the Nogo-N2 was linked to the process of conflict monitoring rather than to inhibition. The topographic Nogo-N2 analyses across 30 electrodes showed that the Nogo distributions of both children and adults were dominated by the commonly reported negative field above medial prefrontal cortex (Bekker et al., 2005; Kiefer et al., 1998; Nieuwenhuis et al., 2003). However, the distributions of children showed extra posterior positivity at bilateral temporal-parietal locations in 6- to 7-year-olds and above medial parietal cortex in 9- to 10-year-olds. Such enhanced posterior activity in the Nogo-N2 interval in children (compared to adults) was reported earlier by Ciesielski et al. (2004) in a similar task. These ERP findings are also supported by developmental functional imaging studies that report activation of larger and more diffuse networks in children or adolescents during performance of tasks requiring cognitive control (for reviews, see Durston and Casey, 2006; Luna and Sweeney, 2004). In Jonkman (2006) the extra posterior activation in children in the Nogo-N2 interval was hypothesized to be associated with increased attentional processing or enhanced attention allocation during conflict monitoring in childhood. The present study had the aim to verify these hypotheses by performing further source analyses on the data.

In prior source localization studies in which Go/Nogo tasks were presented to adult subjects the source of the Nogo-N2 has been repeatedly localized in Anterior Cingulate Cortex (ACC; Bekker et al., 2005; Kiefer et al., 1998; Nieuwenhuis et al., 2003; Van Veen and Carter, 2002a), a brain structure that has been frequently linked to conflict monitoring and response competition in fMRI studies (Botvinick et al., 1999, 2001, 2004; Barch et al., 2001; Braver et al., 2001). To our knowledge, as yet no developmental source localization studies on Nogo-activity have been performed. However, there have been several developmental fMRI studies investigating brain activation differences between children (ranging from 6 to 12 years-old) and adults during performance of a Go/Nogo task (Booth et al., 2003; Bunge et al., 2002a; Casey et al., 1997; Durston et al., 2002; Tamm et al., 2002). These studies have yielded different patterns of Nogo-activation in prefrontal cortex in children and adults, some studies reporting enhanced and others reporting reduced activation in children (compared to adults) in different regions of PFC. Furthermore, congruent with topographical results from Jonkman (2006), in two studies Nogo performance was reported to be more strongly associated with activation of posterior areas in children than adults (Bunge et al., 2002a; Durston et al., 2002). The fact that such Nogo-posterior cortex activations in children were only reported in two out of six developmental fMRI studies might be explained by different fac- tors, an important one being differences in task design. In most of the above-mentioned fMRI studies blocked, instead of event-related, designs (that are more comparable to those used in ERP research) were applied. In fact, the studies that did use an event-related design did report prefrontal as well as posterior activation in children and adults in the Nogo condition. In Durston et al. (2002) adults showed significantly higher activation in the Nogo condition in bilateral ventral PFC, left ACC and left caudate nucleus. Children were reported to have higher Nogo-activation than adults in right DLPFC as well as in right parietal cortex. In Bunge et al. (2002a) the Nogo condition activated multiple prefrontal, temporal and parietal association areas in adults, whereas overall, only small Nogoactivation was seen in children. However, inhibition success was more strongly associated with activation of posterior (parietal, occipital and temporal), instead of prefrontal, areas in children, whereas no activation-inhibition behavior associations were seen in adults. Another important difference between fMRI and ERP studies is that, albeit with considerably lower spatial resolution, it is possible to localize sources on a millisecond time-scale whereas in fMRI studies it is impossible to conclude to what extent the Nogo-activation explains Nogo-N2 or Nogo-P3 activity. This is especially important since Nogo-N2 and Nogo-P3 have been interpreted as reflecting different stages of Nogo processing, respectively, monitoring of response conflict and response inhibition.

In most previous studies, source analysis has been performed on Nogo-Go difference waves (Bokura et al., 2001; Kiefer et al., 1998; Nieuwenhuis et al., 2003; Van Veen and Carter, 2002a). Although the use of difference waves enables one to get rid of activity that is similar in Go and Nogo conditions, the task assignment in the go condition to press a button leads to specific motor and attention related effects on only the go-ERP signal that will also contribute to the Nogo-Go difference wave. Accordingly, in several studies, besides a source in prefrontal cortex additional sources were required to adequately explain the Nogo-N2 distribution. Bokura et al. (2001) for instance reported an additional source in the left thalamus, whereas Nieuwenhuis et al. (2003) reported medial posterior involvement in a task condition with $80 \%$ Nogo trials. To prevent the contribution of such motor or attention processes from the Go condition, in the present study, dipole models were computed directly on the Nogo ERP. However, a complicating factor in localizing sources on the Nogo-wave only is the presence of the fronto-central Nogo-P3 positivity that has been demonstrated by Bekker et al. (2005) to dominate the Nogo-N2 scalp topography and source models in adults. Bekker et al. circumvented this problem by subtracting peak Nogo-P3 activity from the average. It was shown that such corrected Nogo-N2 data yielded better source estimates, i.e. in the vicinity of the ACC, than Nogo-N2 data that were not corrected for P3 activity. Following Bekker et al. (2005) this baseline correction method was also applied in the present study. It was 
expected that, in the adult group, using this subtraction method the Nogo-N2 source would be localized near ACC. In addition, it was expected that application of this baseline correction procedure would also yield better source estimates in children.

Based on topographical data from Jonkman (2006), and the above-mentioned fMRI studies, a frontal source near ACC was expected in all three age groups. Furthermore, based on the presence of additional posterior activity in the Nogo-N2 interval in children in our prior study (Jonkman, 2006) and event-related developmental fMRI studies (Bunge et al., 2002a; Durston et al., 2002), it is expected that the Nogo-N2 distributions of children are explained by the involvement of posterior sources additional to prefrontal sources.

\section{Methods}

\subsection{Subjects}

The present data analyses were performed on the data from exactly the same children and adults that participated in Jonkman (2006). Children were recruited from two different elementary schools and were split up in two groups: a group of 16 children ( 4 boys/12 girls) aged between 6 and 7 years, mean age was 7.1 years $(\mathrm{SD}=0.5)$ and a group of 17 children ( 6 boys/11 girls) aged between 9 and 10 yearsold, mean age 9.6 years $(\mathrm{SD}=0.6)$. The adult group consisted of 17 students from Maastricht University (2 male and 15 female) aged between 19 and 23 years (mean age 21 years, $\mathrm{SD}=1.7$ ) that were paid for participation. Children were rewarded with a present (a toy) after completion of the experiment. The presence of Internalizing or Externalising behavioral problems or attention problems in children were excluded by letting parents fill in a Child Behavior Checklist (CBCL) and excluding all subjects with scores in the clinical or threshold range (for more details, see Jonkman, 2006). Whereas IQ did not significantly differ between the child groups $(p=.44)$, adults had somewhat higher IQ, but IQ did not influence performance differences between groups (see Jonkman, 2006). The present study was approved by a local Ethical Committee and is performed in accordance with the ethical standards laid down in the 1964 Declaration of Helsinki. The caretakers of all children and all adults filled in an informed consent form prior to the start of the study.

\subsection{Procedure}

The entire experimental session lasted 2-2.5 h. The session started with attachment of the electrodes. For measurement of the EEG an electro-cap consisting of 30 tin electrodes was used (Fp1, Fp2, F7, F3, Fz, F4, F8, FT7, FC3, FCz, FC4, FT8, T7, C3, Cz, C4, T8, TP7, CP3, CPz, CP4, TP8, T5, P3, Pz, P4, T6, O1, Oz, O2), nomenclature according to American Electroencephalographic society (1991). All electrodes were referenced to the left mastoid, one of the electrodes in the electro-cap $(\mathrm{FPz})$ was used as ground. The vertical EOG was recorded from infra-orbital and supraorbital tin electrodes placed in line with the pupil of the left eye, for measurement of the horizontal EOG two tin electrodes were attached to the outer canthi of both eyes. All electrode impedances were kept below $5 \mathrm{k} \Omega$, with exception of EOG electrodes that, in some children, were kept between 5 and $18 \mathrm{k} \Omega$ due to low tolerance of impedance reducing methods (scratching) in the face. During electrode attachment children watched cartoons. The experimental session started by installing the subjects in an electrically shielded and sound-proof cabin. The subjects were monitored outside the cabin by a camera system, and communication was possible via an intercom. The subjects sat in front of a $17^{\prime \prime}$ VGA monitor with their eyes aligned to the centre of the screen at a distance of approximately $50 \mathrm{~cm}$. The participants were instructed to minimise eye blinks and refrain from making movements during task performance. The experimental session started when all tasks were practiced until a pre-determined performance criterion $(80 \%$ correct detections and less than $10 \%$ false alarms) was reached. On demand, some parents sat behind their children in the cabin during the experiment. Signal acquisition was accomplished using Neuroscan synamps amplifiers and Neuroscan software (version 4.1). EEG and EOG signals were continuously sampled at $250 \mathrm{~Hz}$ and digitally filtered online with a high-pass filter of $0.05 \mathrm{~Hz}$ and a low-pass filter of $50 \mathrm{~Hz}$.

\subsection{Task}

The CPT-AX task consisted of a sequence of 11 different letters (A, B, C, D, E, F, G, H, J, L and X). The letters were white and were presented one by one between two vertical bars at the centre of the screen on a black background. The vertical bars and the letters had a height of $2 \mathrm{~cm}$ and letters had a width of $1.5 \mathrm{~cm}$. Subjects were seated approximately $50 \mathrm{~cm}$ in front of a $17^{\prime \prime}$ VGA monitor.

The task instruction to the subjects was to press a button with their right hand when the letter $\mathrm{X}$ appeared, but only when it was preceded by the A (A-X sequence, Go condition). When an $\mathrm{A}$ is followed by another letter the prepared response had to be inhibited (A-not-X, Nogo condition). The task was administered in four separate blocks of 124 trials each, consisting of $24 \mathrm{X}$ and $24 \mathrm{~A}$ stimuli (of which 12 A's and 12 X's were part of the A-X (Go) sequence, 12 A's were part of A-not-X (Nogo) sequences and 12 X's were presented alone, without a preceding A). Thus, the occurrence of Go and Nogo trials was equiprobable (in 10\% of all presentations), since A's were presented in both Go and Nogo sequences and X's were presented in Go sequences and alone, total probability of A's and X's was comparable $(20 \%)$. The remaining 76 trials consisted of 6 trials of letters B, C, D, E, F, G, J, L (total 48 trials) and 28 presentations of the letter $\mathrm{H}$. The letter $\mathrm{H}$ was presented more often to keep the probability (23\%) comparable to that of the letters A and X. A total trial lasted 
$1650 \mathrm{~ms}$, with a stimulus duration of $150 \mathrm{~ms}$ and a fixed interstimulus-interval (ISI) of $1500 \mathrm{~ms}$. Before the start of the experimental session the task was practiced (each subject received 25 practice trials) and all subjects scored at or above $80 \%$ correct responses and had less than $10 \%$ false alarms during the practice session.

\subsection{Dipole source analyses}

Iso-potential contour maps and instantaneous dipole models were determined using the BESA program (version 5.1). Instantaneous dipole models were computed on the grand-average Nogo-waveforms in the latency window where the Nogo-N2 was maximal in the three age groups. The head-model used was a four-shell ellipsoidal head model with default values of bone thickness $(7.0 \mathrm{~mm})$ ) and bone conductivity (0.0042: relative value compared to the conductivity of the CSF) for the adult subjects. Due to known or estimated differences in bone thickness (Koenig et al., 1995; Wiart et al., 2005) and bone conductivity in children, these values were adjusted to $5.7 \mathrm{~mm}$ and 0.012 for the $9 / 10$ year-old children and to $5.2 \mathrm{~mm}$ and 0.016 for the $6 / 7$ year-old children. The parameters used in the present study were obtained by "adjusting them empirically using somatosensory evoked potentials and magnetic fields in several children" (M. Scherg, personal communication).

Dipole pairs were fitted with locations and orientations constrained to be mirror-symmetrical. Source models were computed in an 8-ms window around the Nogo-N2 peak latency determined at FCz; at 256, 292 and $316 \mathrm{~ms}$, respectively, for adults, 9/10 year-old children and 6/7 year-old children. For each age group separately, the best fitting source model on the grand average Nogo-N2 distribution was computed. During the fitting process there was an energy constraint of $20 \%$ to reduce the probability of interacting dipoles. The procedure was as follows: first one dipole pair was fitted (following the hypothesis of medial frontal cortex activity underlying the Nogo-N2) by randomly varying starting positions. Source models were only accepted when being stable across varying starting positions and when explaining $95 \%$ or more of the variance. If according to these criteria, the data could not adequately be explained by the first source pair, a second bilateral dipole pair was added to the model, iteratively adapting its location. During the fit process, orientation and location parameters of the first and second dipole pairs were allowed to vary. All reported dipole solutions were stable across different seeding locations.

As explained more elaborately in the introduction, the Nogo-N2 scalp topography is dominated by a succeeding P3 positivity that "lifts" the N2 (Bekker et al., 2005) and this positivity has been shown to dominate the Nogo-N2 topography in adults and children (Bekker et al., 2005; Jonkman, 2006), causing a shift of the Nogo-N2 source to a more central-posterior location. To check whether we could replicate these results from the latter study, we first fitted a single dipole pair on the Nogo-N2 topography that was not corrected for the Nogo-P3 positivity. The second step was to compute source models after subtracting the Nogo-P3 activity from the data (following Bekker et al., 2005). To eliminate Nogo-P3 activity, first, a $40 \mathrm{~ms}$ interval around the Nogo-P3 peak was determined at $\mathrm{FCz}$ in each group in the grand average waveform; at 368, 392 and $464 \mathrm{~ms}$ for adults, 9/10 year-old children and 6/7 year-old children, respectively. Then the mean activity in this 40-ms interval was subtracted from the Nogo-N2 activity. After applying this subtraction procedure, for each age group the best fitting source model was computed in the mentioned Nogo-N2 windows, following the above-mentioned procedure.

To statistically analyze group differences with regard to dipole moments, source models of the group averages were applied to individual baseline-corrected Nogo ERPs. The source model was optimized at the individual latency at which the $\mathrm{FCz}$ negativity was maximal, with location parameters fixed and orientation parameters free. Overall group effects with respect to dipole moments were examined by performing a repeated measures ANOVA with within-subjects factor Hemisphere (left/right) and between-subjects factor Group (6/7 years, 9/10 years, and adults). Further differences between the groups with respect to dipole moments were tested by applying pairwise comparisons. For statistical analysis, a significance level of $p<.05$ was adopted.

\section{Results}

\subsection{Behavioral results}

Behavioral results will be only briefly reviewed here, for an elaborate discussion of behavioral results the reader is referred to the Jonkman (2006) paper. The 6- to 7-yearold children were shown to be less attentive than 9- to 10 -year-old children and adults (older children also having worse performance than adults) by having a lower percentage of detected targets and a slower and more variable response pattern. No differences between the youngest and older children were reported regarding the percentage of Nogo false alarms, but both groups had significantly lower Nogo accuracy than adults.

\subsection{Source analysis results}

Grand-average go and Nogo ERPs in all three age groups at FCz and Pz electrode sites are presented in Fig. 1.

First, source models were computed for the original Nogo-N2 data that were not corrected for overlapping P3 activity. All source models and iso-potential spline maps were derived at peak latency of the average-referenced N2 wave of successfully inhibited Nogo-trials. For all groups, the obtained single bilateral source model yielded acceptable R.V. (2.2\%, $2.2 \%$ and $3.8 \%$ for adults, $9 / 10$ year-olds and $6 / 7$ year-olds, respectively) with source pairs located in 


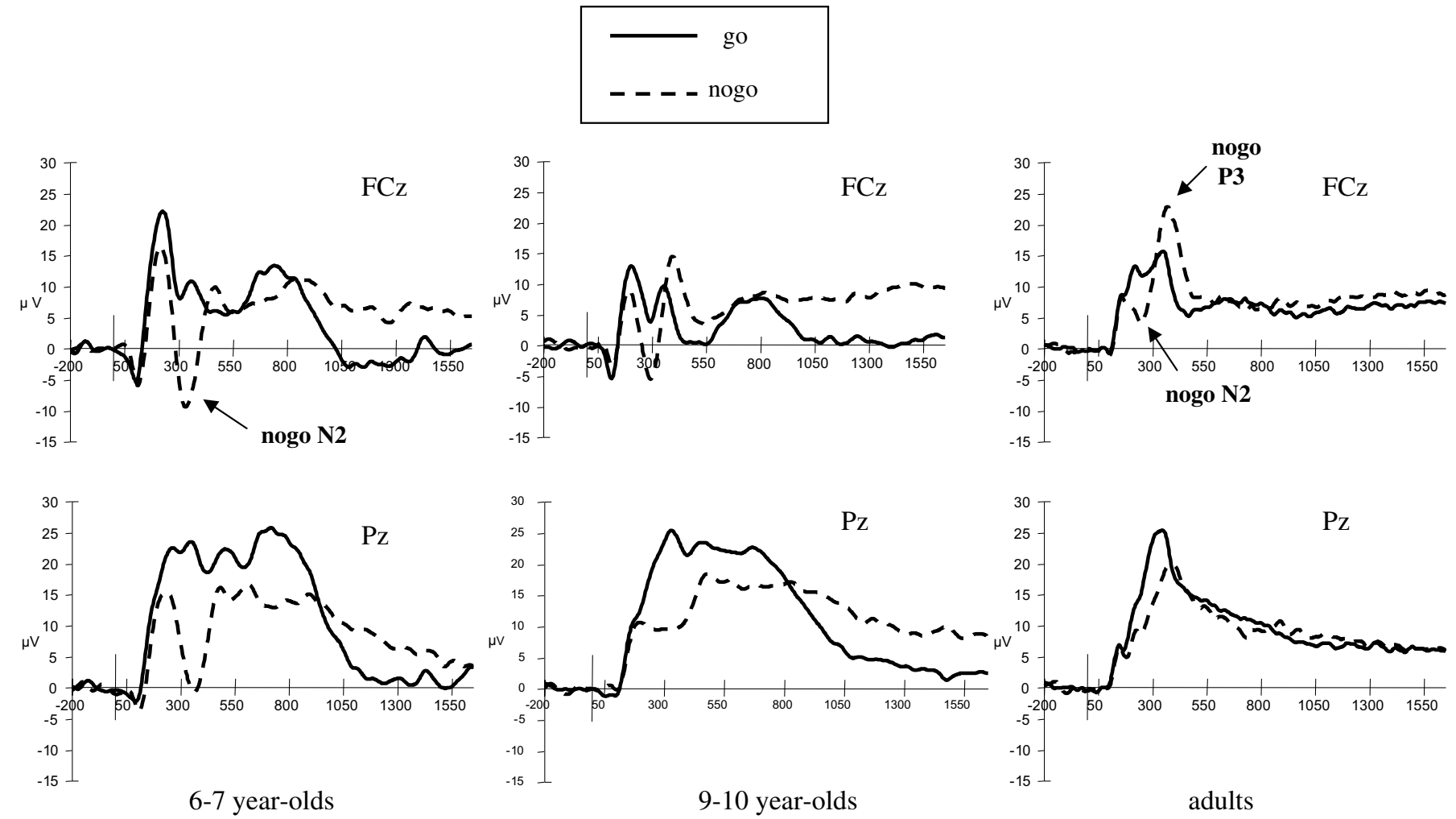

Fig. 1. Go and Nogo grand-average ERP-waves at the FCz and Pz electrodes for all age-groups.

central and precentral brain regions. Source pairs were, however, located deeper in the brain in children compared with adults, with a more posterior location for the youngest children (see Fig. 2). Furthermore, although the source pair appeared to be oriented to the parietal lobe in adults, in children the orientation of the dipoles turned towards the back of the head. It is clearly visible in Fig. 2 that a posterior positivity is dominating the scalp distribution at Nogo-
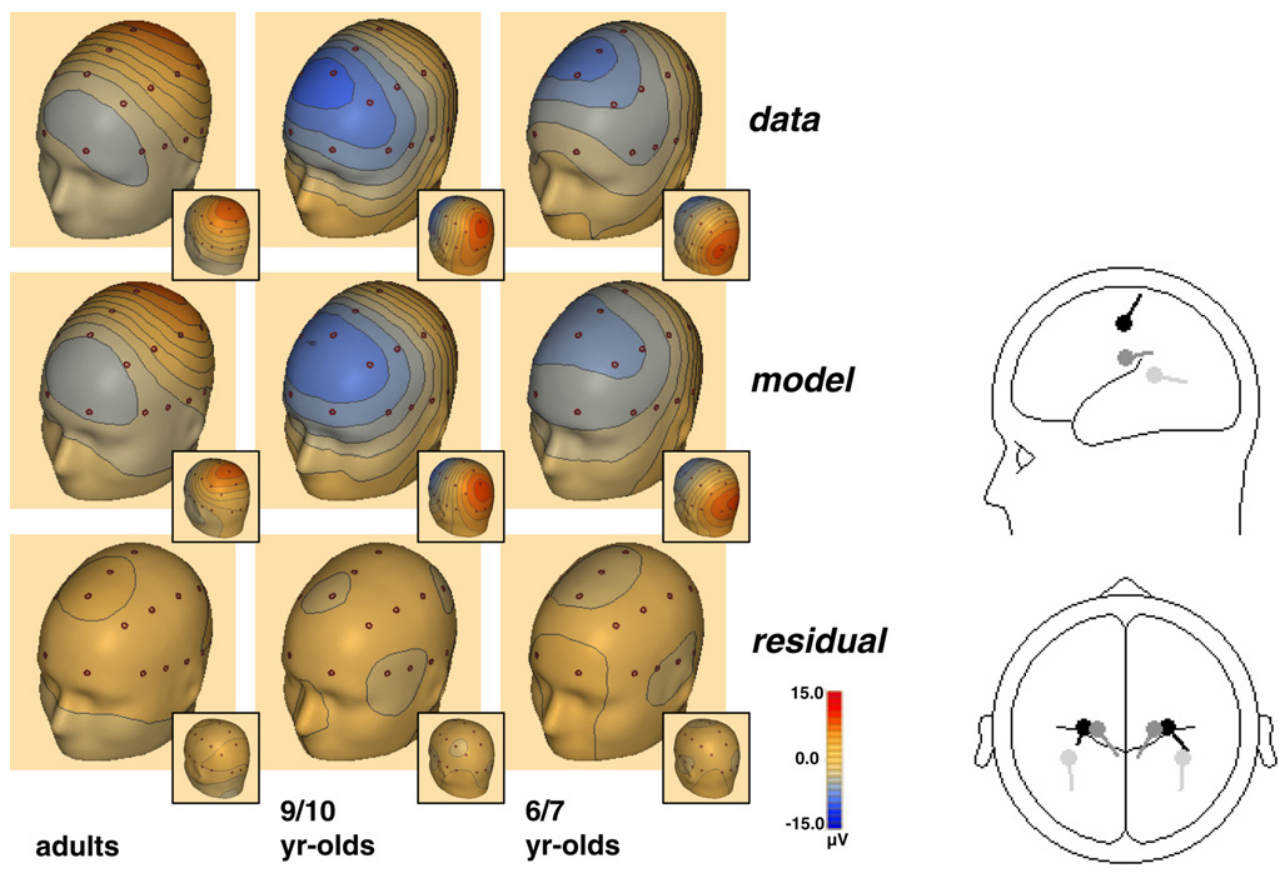

Fig. 2. Original (only corrected for pre-stimulus baseline) iso-potential spline maps and source models derived at Nogo-N2 peak latency of the averagereferenced group-averages for all age groups. Iso-potential spline maps represent the potential distribution of the data, the model and the residual (activity that is not explained by the source model). Source models of the adults, $9 / 10$ year-olds and 6/7 year-olds are presented in black, dark-grey and light-grey, respectively. Dots represent source location, whereas the line represents source orientation. 
Table 1

Cartesian coordinates $(x, y, z)$ of the source locations in adults, 9/10 yearold children and $6 / 7$ year-old children

\begin{tabular}{lrrr}
\hline & $x$ & $y$ & $z$ \\
\hline Original data & & & \\
Adults & 0.31 & 0.01 & 0.58 \\
6/7 year-olds & 0.43 & -0.21 & 0.21 \\
9/10 year-olds & 0.22 & -0.01 & 0.33 \\
Nogo-P3 corrected data & & & \\
Adults & 0.26 & 0.43 & 0.28 \\
6/7 year-olds & & & 0.25 \\
$\quad$ Anterior & 0.11 & 0.30 & -0.10 \\
$\quad$ Posterior & 0.49 & -0.55 & 0.45 \\
9/10 year-olds & & & 0.38 \\
$\quad$ Anterior & 0.16 & -0.25 & 0.32 \\
$\quad$ Posterior & 0.51 & & \\
\hline
\end{tabular}

Since bilateral source pairs were symmetrically constrained with respect to location, only coordinates of the source in the right hemisphere were reported. Anterior, frontal; Posterior, parietal/occipito-temporal.

N2 peak latency in adults (see Bekker et al., 2005 for similar results) and children. Consequently, the source models derived at this Nogo-N2 peak latency seem to explain in essence this posterior positive activity (see Table 1 for $x$, $y, z$ location coordinates of the sources in the different groups).

As was explained earlier, in order to eliminate this posterior positivity on which the Nogo-N2 "rides", a baseline correction was applied around peak latency of Nogo-P3. The spline maps in Fig. 3 demonstrate the successful removal of this positivity from the data in all age groups. For adults, this P3-corrected Nogo-N2 scalp distribution was adequately explained by a bilateral dipole pair
Table 2

Mean (M) dipole moments and corresponding standard errors (SE) of the frontal pair of sources of the "Nogo-P3 corrected" source model for all age groups

\begin{tabular}{|c|c|c|c|c|c|c|}
\hline & \multicolumn{2}{|c|}{$6 / 7$ year-olds } & \multicolumn{2}{|c|}{ 9/10 year-olds } & \multicolumn{2}{|c|}{ Adults } \\
\hline & $\bar{M}$ & SE & $\bar{M}$ & $\mathrm{SE}$ & $\bar{M}$ & SE \\
\hline Left frontal source & 79.0 & 9.4 & 72.6 & 9.2 & 91.6 & 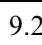 \\
\hline Right frontal source & 77.5 & 9.2 & 59.3 & 9.0 & 98.7 & $9 .($ \\
\hline
\end{tabular}

$(\mathrm{R} . \mathrm{V} .=1.8 \%)$ that was situated medially in the frontal brain near ACC, with orientation towards the medial orbital cortex. Since a bilateral source pair in frontal cortex did not adequately explain the data for the two groups of children (R.V. $\sim 15 \%$ in both groups), a bilateral dipole pair was added to the model, allowing location and orientation parameters of the first acquired single source pair to vary. The R.V. of this new model (see Fig. 3) was $0.5 \%$ for the $6 /$ 7 year-old children and $0.3 \%$ for the $9 / 10$ year-old children. The anterior source pair was located medially in the frontal brain near ACC in both groups of children, at positions close to the adult source pair. The frontal sources in children had also similar orientation as those in adults (see Table 1 for location coordinates in all groups). However, the posterior source pair was situated laterally in the parietal lobe in 9/10 year-old children, whereas it was shifted towards the occipital lobe in 6/7 year-olds. Because adults did not show such posterior sources, only dipole moments of the frontal source pair were statistically analyzed between the three age groups. Although adults appear to have slightly stronger activation of the frontal source pair than both $6 / 7$ and $9 / 10$ year-old children (see Table 2), fur-

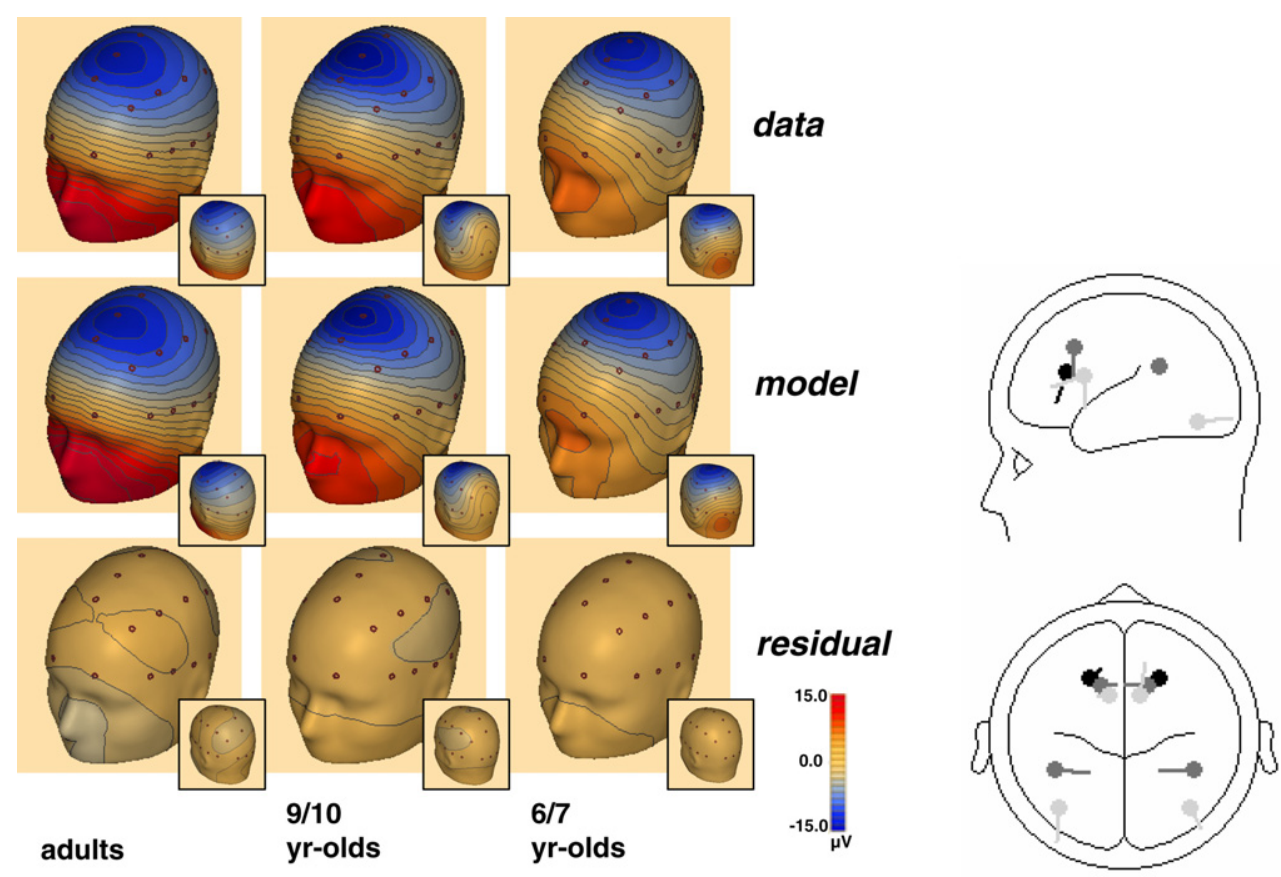

Fig. 3. Source models and iso-potential spline maps of 6/7 year-old children (light-grey), 9/10 year-old children (dark-grey) and adults (black), derived at Nogo-N2 peak latency after correcting the Nogo-ERP for overlapping Nogo-P3 positivity. 
ther testing of a significant group main effect by computing group contrasts $(F(2,47)=3.5, p<.05)$ did not lead to significant differences between the groups. No Hemisphere or Hemisphere $\times$ Group effect was found.

\section{Discussion}

The present study applied source localization procedures to developmental ERP data that were obtained in a prior study by Jonkman (2006) to verify the conclusion that during the Nogo-N2 children activated frontal as well as posterior cortex whereas adults mainly activated frontal cortex. Source models obtained in the present study confirm the presence of a frontal source generating Nogo-N2 activity in both children and adults. The location of this frontal source is in medial frontal cortex and corresponds with locations in the vicinity of ACC, reported in adults in previous source localization studies (Bekker et al., 2005; Nieuwenhuis et al., 2003; Van Veen and Carter, 2002a,b). Whereas medial frontal activation in the Nogo condition in children has been reported before in developmental fMRI studies, the present ERP study demonstrates that this activation occurs as early as $200-350 \mathrm{~ms}$ after presentation of the Nogo stimulus in both children (starting from 6 to 7 years of age) and adults. The strength of activation of this frontal source appeared to be statistically similar in all age groups. This is in contrast with several fMRI studies reporting enhanced activation of medial frontal cortex in children (compared to adults) in the Nogo condition (Booth et al., 2003; Bunge et al., 2002a; Tamm et al., 2002). Note however that due to its low temporal resolution, fMRI results are not comparable to our source localization results that are computed in the specific Nogo-N2 time interval. On the basis of the fMRI results it is not possible to link activation patterns to specific processes like conflict monitoring or response inhibition, whereas ERP studies have yielded convincing evidence that in adults and children the Nogo-N2 is associated with the process of conflict monitoring (Donkers and Van Boxtel, 2004; Jonkman, 2006; Nieuwenhuis et al., 2003; Van Veen and Carter, 2002a).

In addition to the medial frontal source, in both $6 / 7$ and 9/10 year-old children an additional posterior source pair was necessary to explain their Nogo-N2 scalp distribution with acceptable residual variance $(<5 \%)$. Earlier topographic analyses yielded differences in frontal-parietal activation in the Nogo-N2 interval between children and adults (Jonkman, 2006). In this study, the youngest children were reported to have larger Nogo-N2 effects at both frontal and parietal electrodes than 9- to 10-year-olds and adults (see Fig. 1), but Nogo-N2 amplitudes were larger at frontal than parietal sites in all groups. The topographic analyses furthermore showed that the Nogo-N2 topography of 6- to 7-year-old children differed from that of 9to 10-year-olds and adults by showing significantly enhanced positivity at bilateral temporal-parietal electrode sites (TP7 and TP8). The topography of 9- to 10-year-olds was marked by enhanced medial parietal positivity in comparison to younger children and adults. The present source results supported these findings by the localization of parietal sources (additional to frontal) in the oldest children, whereas posterior sources in the youngest children shifted to more temporal-occipital locations.

Several explanations may be given for the higher activation of posterior parietal or temporal-occipital areas in the Nogo-N2 interval in, respectively 9-10 and 6-7 year-old children as compared to adults. First, in an event-related fMRI study, Bunge et al. (2002a) also reported that activity in a subset of posterior areas (in bilateral parietal, bilateral middle temporal and bilateral occipital cortices) was positively associated with effective response inhibition in the Nogo-condition in 8- to 12- year-old children, but not in adults. A possible explanation for this might be that children revert to lower-order processing in order to compensate for immaturity of higher order processing networks including prefrontal cortex. In this context, parietal cortex has been suggested to be involved in the activation of learned stimulus-response (S-R) associations (Bunge et al., 2002b), whereas occipito-temporal areas are involved in even more basic processing, such as stimulus identification. Perhaps older children reactivate these $\mathrm{S}-\mathrm{R}$ associations in order to check whether a response is appropriate or not, whereas younger children revert to more enhanced basic processing of the stimulus to optimize cognitive control (monitoring of conflict or inhibiting a response). Supporting the present data, several developmental fMRI studies indicate that children recruit more elaborate networks, including posterior areas, than adults when having to inhibit motor responses that conflict with the current response set (Bunge et al., 2002a; Durston et al., 2002). Recently, by using a longitudinal design, Durston et al. (2006) showed that posterior involvement even diminishes between 9 and 11 years of age. At the age of 9 children showed enhanced activity in frontal (DLPFC) and posterior (posterior cingulate, right superior temporal gyrus) areas during Nogo performance compared to when they were 11 years of age. Reduced activity with age was only seen in ventral frontal cortex, the only area in which activation correlated with Nogo accuracy. Such shifts from diffuse to focal cortical activation have been suggested to be associated with synaptic pruning and myelination in combination with strengthening of relevant connections (Luna and Sweeney, 2004; Durston and Casey, 2006). Structural imaging studies have indicated linear increases in white matter up to age 20, whereas non-linear developmental changes have been described for cortical grey matter (reviewed in Toga et al., 2006). Gogtay et al. (2004) revealed first grey-matter losses around 4-8 years of age in dorsal parietal, particularly primary sensorimotor regions, whereas in parietal lobes loss occurred not until adolescence (11-13 years). Giedd et al. (1999) showed grey matter increases in frontal and parietal lobes in pre-adolescence, peaking at 12 years for frontal and parietal lobes, losses occurring only thereafter. These maturational pat- 
terns described for sensori-motor and parietal regions might explain the involvement of these areas in cognitive control in children, although it is as yet unclear how. It might be that only after pruning and myelinisation effective communication between areas or within one area (prefrontal cortex) is possible, reducing the necessity of activating a diffuse network of brain areas. In a recent DTI study radial diffusities along frontostriatal fibers during performance of a Go/Nogo task were shown to be more restricted in adults than in 9-year-old children (Liston et al., 2006a). Although the age groups did not differ in Nogo accuracy, on the basis of correlations between Go-RT performance and DTI changes the authors concluded that refined connectivity is contributing to improvements in cognitive control in adults.

Another explanation for the higher activation of posterior sources in children might be given in the light of the Nogo-N2 as being a reflection of conflict processing. On the basis of behavioral and ERP findings Jonkman (2006) concluded that the enlarged Nogo-N2 that was present in especially 6- to 7-year-old children most likely indicates that children experience a higher level of conflict in the Nogo condition. If this conclusion is valid, the different source activation patterns underlying the Nogo-N2 in children and adults might have to do with the type of conflict that children and adults experience. In a recent eventrelated fMRI study, Liston et al. (2006b) demonstrated that in adults, different types of conflict evoke different patterns of brain activation; posterior parietal cortices were sensitive to conflict manipulations at the stimulus level, whereas ACC was involved in manipulations of conflict at response level. Although these different levels of conflict were not explicitly manipulated in the present study, the recruitment of both frontal and parietal sources by $9 / 10$ year-old children might be indicative of the experience of conflict at both stimulus and response level, whereas adults only experience response conflict. Furthermore, 6/7 yearold children might experience conflict at even more basic stimulus-related levels than the 9/10 year-old children, since in this group sources were localized in the occipitaltemporal cortex. Such hypotheses should however be further investigated in a developmental study in which different types of conflict are manipulated.

To avoid the influence of confounding attention or motor effects on our source model, following Bekker et al. (2005) we computed source models underlying Nogo-N2 activity directly on the Nogo-wave and applied a baseline correction for the influence of overlapping positivity in the Nogo-N2 interval. The results from Bekker et al. were replicated in the present study by showing that without correction, Nogo-N2 scalp topography and source models were dominated by a positive field in both adults and children, causing the source to shift to a central location in the vicinity of the precentral sulcus, instead to a location near ACC. Jonkman (2006) already reported that subtraction of overlapping Nogo-P3 led to Nogo-N2 topographies with a clear negative field above frontal cor- tex in both adults and children. The present study confirmed these results by showing that after subtraction of the Nogo-P3 positivity, the Nogo-N2 source indeed shifted to a medial frontal location in adults, replicating Bekker et al. (2005). But additionally, the present results show that even in children, who do not show such profound Nogo-P3 activity as adults, the correction procedure caused a shift of the Nogo-N2 source to a frontal location similar to that of adults. As discussed above, still an extra posterior source pair was necessary for adequate modelling in both child groups, but these sources were localized in the vicinity of parietal or occipital-temporal areas.

In the present study bone thickness and conductivity values were adapted in the models of children. Differences in skull thickness between children and adults are relatively well known and (e.g. Koenig et al., 1995) have relatively little influence on source model location parameters. Whereas there is debate about the exact differences in skull conductivity (relative to CSF conductivity) between children and adults, it is assumable that they are different. In the present study we used conductivity values for children that were obtained by "adjusting them empirically using somatosensory evoked potentials and magnetic fields in several children" (M. Scherg, personal communication). Thus, although these values are presumably not $100 \%$ accurate, they seem at least to be a good estimate. Vanrumste et al. (2000) showed the importance of having a good estimate of skull conductance; underestimation of skull conductivity was shown to lead to maximum location errors in depth of the source of 33 and $28 \mathrm{~mm}$, respectively, for 32 or 53 electrodes with dipoles fitted close to the skullbrain border. The $z$-location of the frontal source in the three groups in the present study and, the fact that the $z$-location was comparable between adults and the youngest children (even though the latter had the highest conductivity values) give further confidence in the chosen conductivity parameters and thus the dipole moment analyses. Another methodological issue is the spatial accuracy of the reported source models. In the present study we used a limited electrode configuration consisting of 30 electrodes. It has been suggested that at least 60 electrodes are needed to correctly sample the scalp electric fields (Michel et al., 2004). However, by implanting sources at different locations in the brain, Krings et al. (1999) demonstrated that, using a 4-shell spherical head model, mean location error was highest in the vertical plane and varied between 13 and $17 \mathrm{~mm}$ when using, respectively, 41 or 21 electrodes. We believe that such spatial resolution is high enough to justify the current source location interpretations.

In conclusion, the present results indicate a source in medial frontal cortex to be responsible for generating Nogo-N2 activity in both adults and children. However, contrary to adults, children also recruit additional posterior areas in this Nogo-N2 window. Although such enhanced activation of posterior brain areas in children has also been reported in previous fMRI studies, to our 
knowledge the present study is the first EEG-source localization study showing that this posterior activation in children already occurs at a very early level of processing at approximately $200-350 \mathrm{~ms}$ post-stimulus (in the Nogo-N2 window). Since children showed lower inhibition accuracy than adults, the enhanced posterior activation in children is suggested to be caused by enhanced attentional demands or enhanced conflict experience.

\section{References}

Barch DM, Braver TS, Akbudak E, Conturo T, Ollinger J, Snyder A. Anterior cingulate cortex and response conflict: effects of response modality and processing domain. Cereb Cortex 2001;11:837-48.

Bekker EM, Kenemans JL, Verbaten MN. Source analysis of the N2 in a cued Go/NoGo task. Brain Res Cogn Brain Res 2005;22:221-31.

Bokura H, Yamaguchi S, Kobayashi S. Electrophysiological correlates for response inhibition in a Go/NoGo task. Clin Neurophysiol 2001;112:2224-32.

Booth JR, Burman DD, Meyer JR, Lei Z, Trommer BL, Davenport ND, et al. Neural development of selective attention and response inhibition. Neuroimage 2003;20:737-51.

Botvinick M, Nystrom LE, Fissell K, Carter CS, Cohen JD. Conflict monitoring versus selection-for-action in anterior cingulate cortex. Nature 1999;402:179-81.

Botvinick MM, Braver TS, Barch DM, Carter CS, Cohen JD. Conflict monitoring and cognitive control. Psychol Rev 2001;108:624-52.

Botvinick MW, Cohen JD, Carter CS. Conflict monitoring and anterior cingulate cortex: an update. Trends Cogn Sci 2004;8:539-46.

Braver TS, Barch DM, Gray JR, Molfese DL, Snyder A. Anterior cingulate cortex and response conflict: effects of frequency, inhibition and errors. Cereb Cortex 2001;11:825-36.

Bunge SA, Dudukovic NM, Thomason ME, Vaidya CJ, Gabrieli JD. Immature frontal lobe contributions to cognitive control in children: evidence from fMRI. Neuron 2002a;33:301-11.

Bunge SA, Hazeltine E, Scanlon MD, Rosen AC, Gabrieli JD. Dissociable contributions of prefrontal and parietal cortices to response selection. Neuroimage 2002b;17:1562-71.

Casey BJ, Trainor RJ, Orendi JL, Schubert AB, Nystrom LE, Giedd JN, et al. A developmental functional MRI study of prefrontal activation during performance of a go-no-go task. J Cogn Neurosci 1997;9:835-47.

Ciesielski KT, Harris RJ, Cofer LF. Posterior brain ERP patterns related to the go/no-go task in children. Psychophysiology 2004;41:882-92.

Donkers FC, Van Boxtel GJ. The N2 in go/no-go tasks reflects conflict monitoring not response inhibition. Brain Cogn 2004;56:165-76.

Durston S, Casey BJ. What have we learned about cognitive development from neuroimaging? Neuropsychologia 2006;44:2149-57.

Durston S, Davidson MC, Tottenham N, Galvan A, Spicer J, Fossella JA, et al. A shift from diffuse to focal cortical activity with development. Dev Sci 2006;9:1-8.

Durston S, Thomas KM, Yang Y, Ulug AM, Zimmerman RD, Casey BJ. A neural basis for the development of inhibitory control. Dev Sci 2002;5:F9-F16.

Falkenstein M, Hoormann J, Hohnsbein J. ERP components in Go/Nogo tasks and their relation to inhibition. Acta Psychol 1999;101:267-91.
Fallgatter AJ, Strik WK. The NoGo-anteriorization as a neurophysiological standard-index for cognitive response control. Int J Psychophysiol 1999;32:233-8.

Giedd JN, Blumenthal J, Jeffries NO, Castellanos FX, Liu H, Zijdenbos A, et al. Brain development during childhood and adolescence: a longitudinal MRI study. Nat Neurosci 1999;2:861-3.

Gogtay N, Giedd JN, Lusk L, Hyashi KM, Greenstein D, Vaituzius AC, et al. Dynamic mapping of human cortical development during childhood and adolescence. Proc Natl Acad Sci 2004;101:8147-79.

Jodo E, Kayama Y. Relation of a negative ERP component to response inhibition in a Go/No-go task. Electroencephalogr Clin Neurophysiol 1992;82:477-82.

Jonkman LM. The development of preparation, conflict monitoring and inhibition from early childhood to young adulthood; a go/nogo ERP study. Brain Res 2006;1097:181-93.

Kiefer M, Marzinzik F, Weisbrod M, Scherg M, Spitzer M. The time course of brain activations during response inhibition: evidence from event-related potentials in a go/no go task. Neuroreport 1998;9:765-70.

Koenig WJ, Donovan JM, Pensler JM. Cranial bone crafting in children. Plast reconstr Surgery 1995;95:1-4.

Krings T, Chiappa KH, Cuffin BN, Cochius JI, Connolly S, Cosgrove GR. Accuracy of EEG dipole source localization using implanted sources in the human brain. Clin Neurophysiol 1999;110:106-14.

Liston C, Watts R, Tottenham N, Davidson MC, Niogi S, Ulug AM, et al. Frontostriatal microstructure modulates efficient recruitment of cognitive control. Cereb Cortex 2006a;16:553-60.

Liston C, Matalon S, Hare TA, Davidson MC, Casey BJ. Anterior cingfulate and posterior parietal cortices are sensitive to dissociable forms of conflict in a task switching paradigm. Neuron 2006b;50:643-53.

Luna B, Sweeney JA. The emergence of collaborative brain function: FMRI studies of the development of response inhibition. Ann N Y Acad Sci 2004;1021:296-309.

Michel CM, Murray MM, Lantz G, Gonzalez S, Spinelli L, Grave de Peralta R. EEG source imaging. Clin Nuerophysiol 2004;115:2195-222.

Nieuwenhuis S, Yeung N, van den Wildenberg W, Ridderinkhof KR. Electrophysiological correlates of anterior cingulate function in a go/ no-go task: effects of response conflict and trial type frequency. Cogn Affect Behav Neurosci 2003;3:17-26.

Tamm L, Menon V, Reiss AL. Maturation of brain function associated with response inhibition. J Am Acad Child Adolesc Psychiatry 2002;41:1231-8

Toga AW, Thompson PM, Sowell ER. Mapping brain maturation. Trends Neurosci 2006;29:148-59.

Vanrumste B, Van Hoey G, Van de Walle R, D'Have M, Lemathieu L, Boon P. Dipole location errors in electroencephalogram source analysis due to volume conductor model errors. Med Biol Eng Comput 2000;38:528-34.

Van Veen V, Carter CS. The timing of action-monitoring processes in the anterior cingulate cortex. J Cogn Neurosci 2002a;14:593-602.

Van Veen V, Carter CS. The anterior cingulate as a conflict monitor: fMRI and ERP studies. Physiol Behav 2002b;77:477-82.

Wiart J, Hadjem A, Gadi N, Bloch I, Wong MF, Pradier A, et al. Modeling of RF head exposure in children. Bioelectromagnetics Suppl 2005; 7:S19-30. 